\title{
CORRIGENDUM
}

\section{Instability of a thin viscous film flowing under an inclined substrate: steady patterns - CORRIGENDUM}

\author{
G. Lerisson, P.G. Ledda, G. Balestra and F. Gallaire
}

doi:10.1017/jfm.2020.396, Published by Cambridge University Press, 30 June 2020

We recently published a paper (Lerisson et al. 2020) on the steady patterns of a thin viscous film flowing under an inclined plane. We have noticed the presence of an error resulting from the non-dimensionalization of the curvature equation (3.7), which is performed with two different characteristic lengths, the inlet thickness $h_{N}$ and the reduced capillary length $\tilde{\ell}_{c}^{*}=\sqrt{\gamma /(\rho g \sin \theta)}$ along the normal and tangential to the substrate directions, respectively. The correct non-dimensional expression of the full curvature reads

$$
\kappa=\frac{\frac{\partial^{2} h}{\partial x^{2}}\left(1+\left(\frac{h_{N}}{\ell_{c}^{*}} \frac{\partial h}{\partial y}\right)^{2}\right)+\frac{\partial^{2} h}{\partial y^{2}}\left(1+\left(\frac{h_{N}}{\ell_{c}^{*}} \frac{\partial h}{\partial x}\right)^{2}\right)-2\left(\frac{h_{N}}{\ell_{c}^{*}}\right)^{2} \frac{\partial h}{\partial x} \frac{\partial h}{\partial y} \frac{\partial^{2} h}{\partial x \partial y}}{\left(1+\left(\frac{h_{N}}{\ell_{c}^{*}} \frac{\partial h}{\partial x}\right)^{2}+\left(\frac{h_{N}}{\ell_{c}^{*}} \frac{\partial h}{\partial y}\right)^{2}\right)^{3 / 2}} .
$$

In Lerisson et al. (2020), the presence of the pre-factor $h_{N} / \ell_{c}^{*}=1 / \tilde{\ell}_{c}^{*}$ was overlooked and we erroneously assumed $\tilde{\ell}_{c}^{*}=1$. This mistake does not influence the results of the linear analyses (where the curvature was linearized, i.e. $\kappa=\frac{\partial^{2} h}{\partial x^{2}}+\frac{\partial^{2} h}{\partial y^{2}}$ ), the experimental measurements, and the comparisons in figures 6,7 , where the difference is invisible to naked eye and does not alter the excellent agreement between numerics and experiments. However, we need to reconsider the claim done on the uniqueness of the rivulet profile in $\S 5$.

The procedure outlined in $\S \S 5.3$ and 5.4 is correct, but it has been performed only for $\tilde{\ell}_{c}^{*}=1$, since in the one-dimensional lubrication equation (5.1) the full expression of the curvature with $\frac{\partial}{\partial x}=0$ is employed. We thus rectify the claim in the last sentence of $\S 5.1$ : the profile is not unique, but there is a family of rivulet profiles. The analysis performed in $\S 5$ with $\tilde{\ell}_{c}^{*}=1$ is repeated for $1<\tilde{\ell}_{c}^{*}<10$, with the results reported in figure 1 of this corrigendum. The rivulet profile indeed depends on $\tilde{\ell}_{c}^{*}$ and tends to the case of linearized curvature (already reported in the original manuscript) as $\tilde{\ell}_{c}^{*} \rightarrow \infty$ (i.e. the thickness goes to zero). These differences are small and hardly visible in the experiments. In the procedure of $\S 5.4$, the equation in the text to retrieve the thickness from $\psi$ is wrong and should be $\mathrm{d} h / \mathrm{d} s=-\tilde{\ell}_{c}^{*} \sin \psi$. As seen in figure 2 of this corrigendum, the resulting family of static pendent rivulets is in excellent agreement with the quasi-steady state of the 

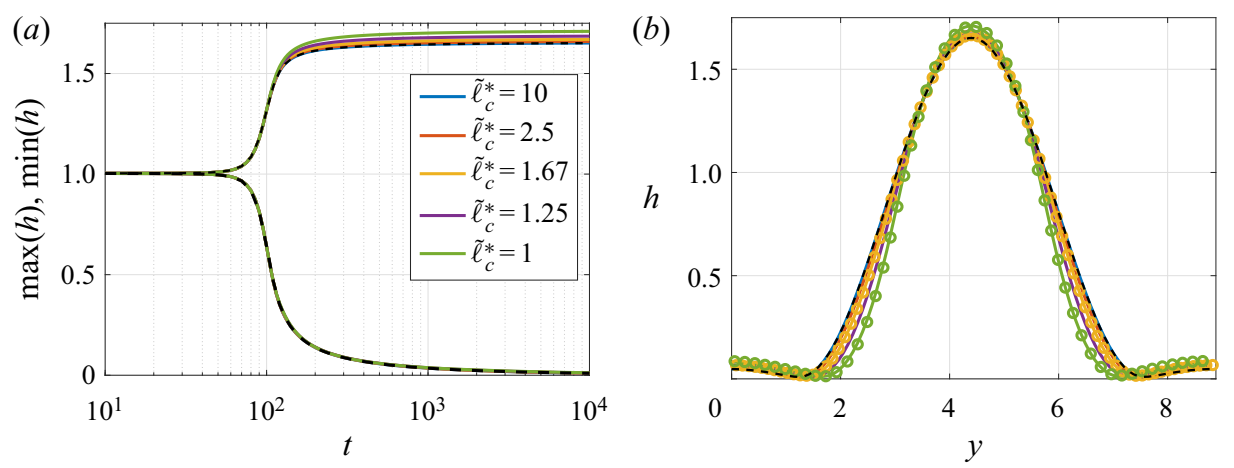

Figure 1. Evolution of the maximum and minimum thickness ( $a$ ) and corresponding rivulet profiles at $t=10^{4}$ (b) for the open flow model of $\S 5.3$ for different $\tilde{\ell}_{c}^{*}$. The circles denote the non-dimensionalized profiles of the two-dimensional Stokes simulations with initial thickness that ensures the correct flow rate. The black dashed lines denote the model with linearized curvature, i.e. $\tilde{\ell}_{c}^{*} \rightarrow \infty$.
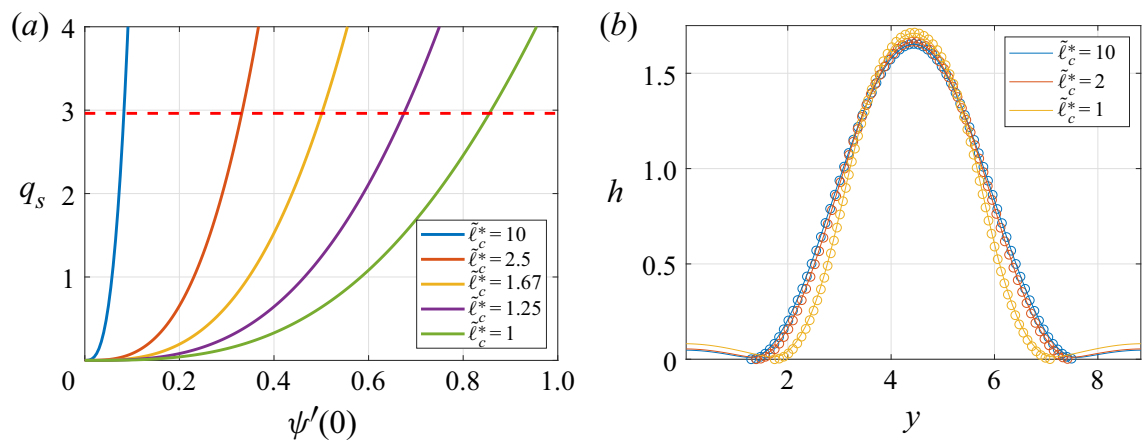

Figure 2. Results of the elastica procedure of $\S 5.4$ for different $\tilde{\ell}_{c}^{*}$. (a) Flow rate $q_{s}$ as a function of the initial curvature $\psi^{\prime}(0)$. The red dashed line denotes the rivulet flow rate to be imposed. (b) Comparison of the open flow model (solid lines) with the elastica one (circles).

central lobe reached in the dynamic calculations. The correct value of the initial curvature $\psi^{\prime}(0)$ depends now on $\tilde{\ell}_{c}^{*}$. We verified the faithfulness of these results with the lubrication equation and Stokes simulations in their dimensional form, the profiles of which were successively non-dimensionalized.

The main message of $\S 5$ remains valid, i.e. the rivulet profile is a one-dimensional drop with imposed normal flow rate and thus a pure equilibrium between surface tension and gravity. However, there is a family of rivulet profiles which depend weakly on $\tilde{\ell}_{c}^{*}$ and tend to the linearized curvature case as $\tilde{\ell}_{c}^{*}$ increases.

Funding. We acknowledge the Swiss National Science Foundation under grant 200021_178971.

Declaration of interests. The authors report no conflict of interest.
Author ORCIDs.
D P.G. Ledda https://orcid.org/0000-0003-4435-8613;
(D) G. Balestra https://orcid.org/0000-0003-0954-5157;
(b) F. Gallaire https://orcid.org/0000-0002-3029-1457. 


\section{Corrigendum}

\section{REFERENCE}

Lerisson, G., Ledda, P.G., Balestra, G. \& Gallaire, F. 2020 Instability of a thin viscous film flowing under an inclined substrate: steady patterns. J. Fluid Mech. 898, A6. 